\title{
Functionality of the GAL4/UAS system in Tribolium requires the use of endogenous core promoters
}

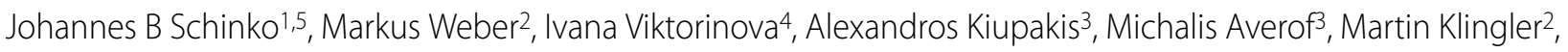 \\ Ernst A Wimmer ${ }^{1}$ and Gregor Bucher*1
}

\begin{abstract}
Background: The red flour beetle Tribolium castaneum has developed into an insect model system second only to Drosophila. Moreover, as a coleopteran it represents the most species-rich metazoan taxon which also includes many pest species. The genetic toolbox for Tribolium research has expanded in the past years but spatio-temporally controlled misexpression of genes has not been possible so far.

Results: Here we report the establishment of the GAL4/UAS binary expression system in Tribolium castaneum. Both GAL4 $\triangle$ and GAL4VP16 driven by the endogenous heat shock inducible promoter of the Tribolium hsp68 gene are efficient in activating reporter gene expression under the control of the Upstream Activating Sequence (UAS). UAS driven ubiquitous tGFP fluorescence was observed in embryos within four hours after activation while in-situ hybridization against tGFP revealed expression already after two hours. The response is quick in relation to the duration of embryonic development in Tribolium - 72 hours with segmentation being completed after 24 hours - which makes the study of early embryonic processes possible using this system. By comparing the efficiency of constructs based on Tribolium, Drosophila, and artificial core promoters, respectively, we find that the use of endogenous core promoters is essential for high-level expression of transgenic constructs.
\end{abstract}

Conclusions: With the established GAL4/UAS binary expression system, ectopic misexpression approaches are now feasible in Tribolium. Our results support the contention that high-level transgene expression usually requires endogenous regulatory sequences, including endogenous core promoters in Tribolium and probably also other model systems.

\section{Background}

The red flour beetle Tribolium castaneum has become established as an important model system, from a group of insects - the Coleoptera - that comprises one fourth of all described animal species [1] including numerous pests (boll weevil, corn rootworm, Colorado potato beetle and Asian longhorn beetle). While the technical amenability of the fruit fly Drosophila melanogaster remains unmatched, there are topics which cannot be readily addressed in the fly. On one hand, evolutionary questions require comparative functional data from several species. Moreover, many processes are derived in Drosophila and data from more insect typical taxa is needed. For instance, segments are specified all at one time in Droso-

* Correspondence: gbucher1@uni-goettingen.de

${ }^{1}$ Ernst Caspari Haus, Georg-August-University Göttingen, Justus-von-LiebigWeg11, 37077 Göttingen, Germany

Full list of author information is available at the end of the article phila (long germ mode) instead of sequential formation that is characteristic of most insects (short germ mode); embryonic legs do not develop in Drosophila while insect larvae usually do have functional walking appendages; extraembryonic membranes are highly reduced and the head is involuted during embryogenesis in Drosophila, resulting in seemingly headless lavae $[2,3]$. On the other hand, certain issues of insect biology cannot be studied in Drosophila because it lacks the respective character. One example are the odoriferous defensive glands that play a crucial role in insect communication and defense but are not found in Drosophila. Hence, there is a need for complementary insect model systems for comparative functional work and for studying processes that are difficult or impossible to study in Drosophila.

Recent development of genetic techniques has rendered Tribolium the second best insect model system. Its genome is sequenced [4], germ line transformation in 
Tribolium is as efficient as in Drosophila, and several marker and transposon systems for gene transfer are available [5-8]. Based on these systems, an insertional mutagenesis system has been established [9] which has been used to generate a collection of enhancer trap and homozygous lethal lines [10]. Most notably, robust RNAi techniques have been established. RNAi can be applied by embryo injection but the systemic uptake of dsRNA also allows injection of female pupae or adults and analysis of the embryonic phenotype in the offspring. Injection of larvae allows to uncover phenotypes during metamorphosis without interfering with earlier (embryonic) gene functions [11-18]. Apparently, all tissues can be targeted by RNAi [14] and the null mutant phenotype can be phenocopied by RNAi in many cases [19].

While knock-down of gene function via RNAi is extremely efficient in Tribolium, spatio-temporally controlled misexpression of genes has not been possible so far. Binary expression systems have the advantage that any gene can be expressed in tissue-specific patterns and at certain developmental stages - depending on the availability of driver lines [20-26]. This allows the study of dominant lethal or sterility inducing genetic constructs because the transgene is only activated when the driver and responder activities are combined. One widely used binary expression system is the GAL4/UAS system, which consists of a driver construct, where expression of the heterologous transactivator GAL4 is driven by an inducible or tissue specific enhancer. In the responder construct, the gene of interest is under the control of the heterologous GAL4-controlled Upstream Activating Sequence (UAS) [20,27-29]. For driver and responder, separate transgenic lines are generated and upon crossing these strains, the gene of interest is expressed in the progeny in the pattern defined by the driver.

GAL4 was identified in the yeast Saccharomyces cerevisiae as a regulator of GAL1, GAL10 and other genes induced by galactose [30,31]. GAL4 regulates transcription by binding to a 17 bp site in the UAS [32]. The GAL4 transactivator consists of two functional domains. The DNA binding domain maps to the first 74 amino acids whereas the activation domain maps to two regions, amino acids 148-196 and 768-881. In the transactivator version GAL4 $\Delta$, the activation domain is directly fused to its DNA binding domain[33,34]. This results in a smaller protein which has been shown to activate reporter gene expression about twice as effectively as the original GAL4 in Drosophila [23].

In the GAL4-VP16 version, the activation domain of GAL4 has been replaced by the highly acidic portion of the herpes simplex virus protein VP16 that activates transcription of immediate early viral genes [35-40]. It was shown that GAL4-VP16 can efficiently activate transcription in mammalian cells [41]. Also in Drosophila GAL4-
VP16 is more active than GAL4, but it has been shown to be less useful compared to GAL4 $\Delta$ given that many insertions of GAL4-VP16 constructs appear to be non-functional [23].

After its inception in the mouse [29], the Gal4/UAS system has been established in Drosophila where it has become a standard technique adapted to diverse uses [42]. One of the numerous extensions of this system is its combination with GAL80, a protein that binds to the carboxy-terminal amino acids of GAL4 and inhibits activation of transcription [23,27-29,42-45]. The GAL4/UAS binary system has been adapted to silkworm Bombyx mori [46] zebrafish [47], Xenopus [48] and Arabidopsis [49]. With this work we establish the GAL4/UAS system in Tribolium. We show that both GAL4A and GAL4VP16 transactivate well, with GAL4 $\Delta$ being slightly more efficient. Importantly, we find that the use of Tribolium endogenous core promoters is essential for efficient expression of transgenes.

\section{Methods \\ Constructs}

All transactivator and responder constructs were stably integrated into the genome by transposition using the piggy Bac vectors $\mathrm{pBac}[3 \mathrm{xP} 3-\mathrm{EGFPafm}], \mathrm{pBac}[3 \times \mathrm{P} 3-\mathrm{ECF}-$ Pafm] [50], pBac[3 $\times$ P3DsRedaf] [6] or pXL-BacII[3 $\times$ P3-EYFPaf], which was generated in a series of minimal piggyBac constructs along with pXL-BacII[3 $\times$ P3-EGFPaf], pXL-BacII[3 $\times$ P3-ECFPaf $]$, and pXL-BacII[3 $\times$ P3DsRedaf] by exchanging the EcoRI-EcoRV fragment of pXL-BacII-ECFP [51] with an EcoRI-NruI fragment carrying the fluorescent marker and the restriction sites $A s c \mathrm{I}$ and FseI [6,50]. However, the integration efficiency of pXL--BacII derivatives appears to be lower than with the other version containing more piggyBac sequence (not shown).Transactivator plasmids: pBac[3 × P3-EGFP;Dmhs-GAL4]; pBac[3 × P3-EYFP;3xP3-GAL4-VP16] [23]; pBac[3xP3-EYFP;3xP3-GAL4A] [23]; pBac[3xP3EGFP;Tc-hsp5'-GAL4-VP16] [GenBank acc no GU452684]; pBac[3 × P3-EGFP;Tc-hsp5'-GAL4] [GenBank acc no GU452683]. Responder plasmids: p[51] Bac[3 × P3-Dsred;UAS-Dm-hsp70TATA-LacZ] [23]: pBac[3 $\times$ P3-DsRed;UAS-Dm-hsp70TATA-Tc'giant]; pBac[3 $\times$ P3-EGFP;gUAS-SCP1-DsRed]; pXL-BacII[3 $\times$ P3-DsRed;UAS-Dm-hsp70TATA-Tc'h-EYFP]; pBac[3 $\times$ P3-DsRed;UAS-Tc-bhsp68-tGFP] [GenBank acc no GU452685]; Heat shock plasmid: pBac[3XP3-DsRed;Dmhsp70-EGFP] [52]. The sequences of those constructs that were functional are available at genbank. Sketches of the constructs are depicted in fig. 1, detailed maps are available from the authors. Dm: Drosophila melanogaster, Tc: Tribolium castaneum; hsp: heat shock promoter; bhsp: basal heat shock promoter/core promoter. 


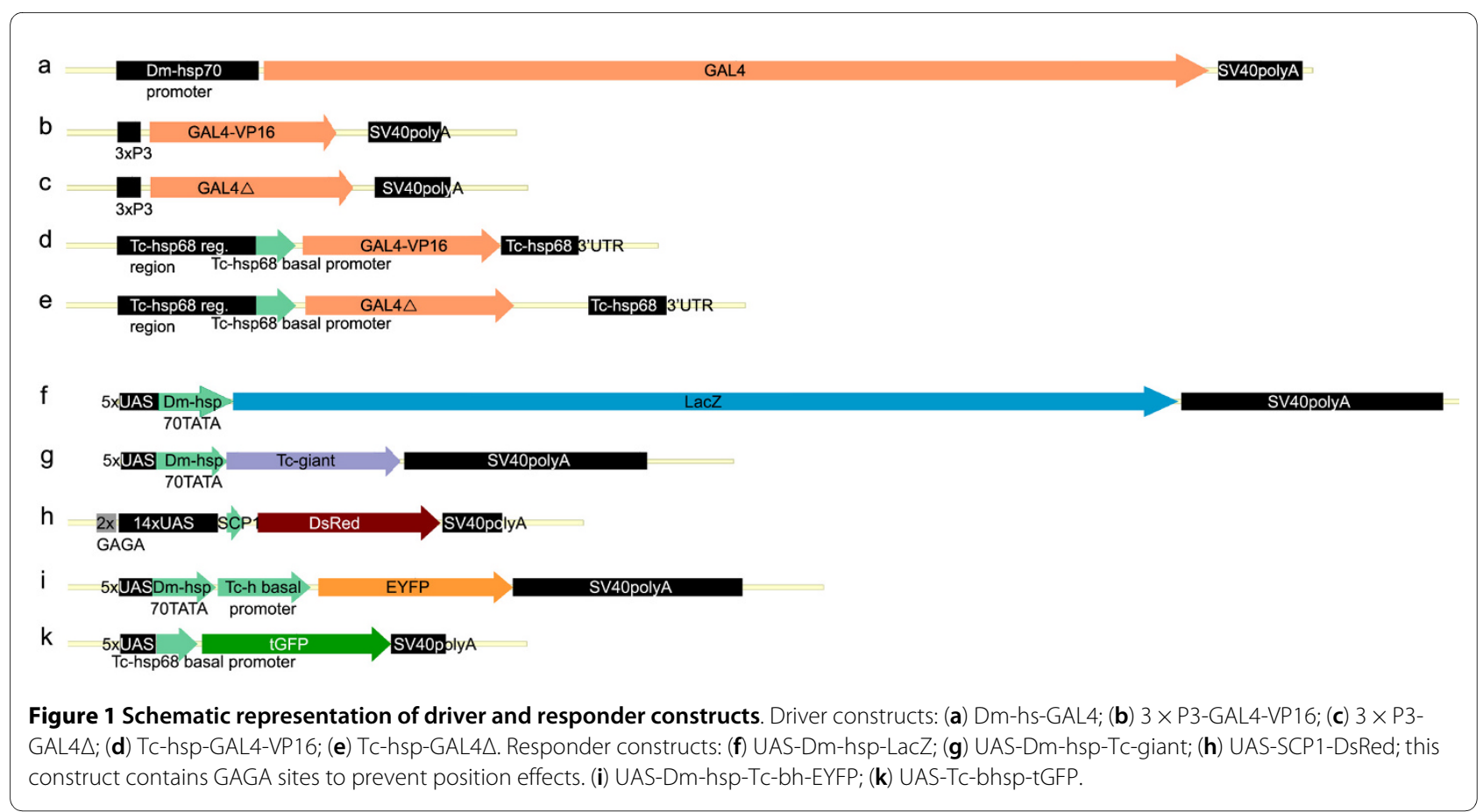

\section{Tribolium stocks and germline transformation}

Tribolium germline transformation was performed according to standard procedure by injecting piggy Bac constructs $(500 \mathrm{ng} / \mu \mathrm{l}$ in injection buffer, i.e. $5 \mathrm{mM} \mathrm{KCl}$, $0.1 \mathrm{mM} \mathrm{KH}_{2} \mathrm{PO}_{4}, 0.1 \mathrm{mM} \mathrm{Na}_{2} \mathrm{HPO}_{4} \mathrm{pH} 6.8$ ) into embryos of the vermillion ${ }^{\text {white }}\left(\nu^{w}\right)$ strain $[5,7,53]$, together with 300 $\mathrm{ng} / \mu \mathrm{l}$ helper plasmid phspBac [54]. A Femto Jet (Eppendorf, Hamburg, Germany) device with pulled and cut borosilicate glass capillaries was used for injections. Injected embryos were kept under humid conditions for two days at $32^{\circ} \mathrm{C} / 90^{\circ} \mathrm{F}$, afterwards transferred to lower humidity and kept at $32^{\circ} \mathrm{C} / 90^{\circ} \mathrm{F}$ until they hatched. Larvae were collected and transferred to full wheat flour. Adult G0 beetles were crossed to $v^{w}$ wild type animals, and transgenic beetles were outcrossed again with $v^{w}$.

\section{Transformation markers and epifluorescence microscopy} As transformation markers, EGFP $[55,56]$ (Clontech Laboratories Inc., Palo Alto, CA), EYFP [57], ECFP [58] as well as the humanized variant DsRed1 [6] were used. The $3 \times$ P3-driven expression pattern of the fluorescent markers was detected in the eyes of T. castaneum using a Leica MZ 16FA fluorescence stereomicroscope with a planachromatic $0.8 \times$ objective (Leica, Wetzlar, Germany). Filter sets used were specifically designed EGFP-LP (Ext. 470/ 40; Emm. 500lp), ECFP-LP (Ext. 425/50; Emm. 460lp), and DsRedwide (Ext. 546/12; Emm. 605/75).

\section{Tribolium crosses}

To activate the binary expression system, adult beetles selected for the dominant markers of the driver and responder lines, respectively, were crossed together and kept for 10 days at $28^{\circ} \mathrm{C} / 81^{\circ} \mathrm{F}$. Within this period of time, sperm from previous matings will be largely replaced by that of the newly added males. Subsequently, the crosses were transferred to fresh flour for egg collections at $32^{\circ} \mathrm{C} /$ $90^{\circ} \mathrm{F}$. Transheterozygous embryos, larvae, pupae or adults were heat shocked and analyzed for tGFP fluorescence.

\section{Heat shock conditions}

Embryonic heat shocks were performed in $1.5 \mathrm{ml}$ Eppendorf tubes in a water bath for 10 minutes at $46^{\circ} \mathrm{C} / 115^{\circ} \mathrm{F}$. Larval, pupal and adult heat shocks were performed in 2 $\mathrm{ml}$ Eppendorf tubes for 20 minutes at $46^{\circ} \mathrm{C} / 115^{\circ} \mathrm{F}$. For activation of the Drosophila constructs in Tribolium the animals were incubated $1 \mathrm{~h}$ at $48^{\circ} \mathrm{C}$ in an incubator.

\section{Detection of tGFP fluorescence in embryos}

After the heat shock, eggs were allowed to recover at $32^{\circ} \mathrm{C}$ (see results section for respective recovery times and then dechorionated under mild conditions in Natriumhypochlorite (1\% DanKlorix), and subsequently aligned on a microscope slide and analyzed for tGFP (Evrogen, Moscow, Russia) fluorescence every hour (Leica MZ 16FA fluorescence stereomicroscope with a planachromatic $0.8 \times$ objective; EGFP-LP filter set, $30 \times$ magnification, $10 \mathrm{sec}$. exposure time). These steps were performed at room temperature.

\section{Detection of reporter gene expression by in situ hybridization}

For comparison of reporter gene expression, eggs from 072 hour collections at $25^{\circ} \mathrm{C} / 77^{\circ} \mathrm{F}$ were heat shocked and 
fixed 11 hours later. Whole-mount in-situ hybridizations were performed as described before [59] with probes of similar size and concentration (tGFP: $770 \mathrm{bp} ; 220 \mathrm{ng} / \mu \mathrm{l}$; DsRed: 740bp, $230 \mathrm{ng} / \mu \mathrm{l}$; eyfp: $790 \mathrm{bp}, 220 \mathrm{ng} / \mu \mathrm{l}$; lacZ: $750 \mathrm{bp}, 230 \mathrm{ng} / \mu \mathrm{l})$. Staining time was the same for all insitu hybridizations.

\section{Results}

Drosophila constructs fail to work consistently in Tribolium Tribolium beetles transgenic for fluorescent proteins under the control of the artificial 3xP3 enhancer-promoter element exhibit strong fluorescence in larval, pupal and adult eyes as well as parts of the nervous system [5]. Similar expression is found in a variety of other arthropods [5,50,60-66]. Moreover, several Drosophila constructs have been shown to work in other species [46,52]. Hence, our first approach to introduce the GAL4/UAS system in Tribolium was to directly transfer constructs tested in Drosophila $[20,23]$ to Tribolium. We used both the transactivator versions GAL4 $\triangle$ and GAL4-VP16 (Fig. $1 b, c)$ driven by the $3 \times \mathrm{P} 3$ enhancer-promoter [23]. Transgenic beetles for each of these constructs were crossed to beetles carrying a responder with lac $Z$ under the control of UAST (Fig. 1f) or UASp (not shown) [20,28]. Due to an endogenous $ß$-galactosidase-like activity in the beetle eyes (not shown) we could not use an enzyme activity assay to detect the lacZ-reporter. By Western blot we did not detect $\beta$-galactosidase in transheterozygotes while Alpha-tubulin was detected. The positive control from an extract of $D$. melanogaster heads expressing lac $Z$ via a functional LexAGAD/(LL)4 system [23] was readily detected (Additional file 1A). We also tested a responder construct with Tc-giant [67] under the control of UAST (Fig. 1g) with a driver consisting of Gal4 under the control of the Drosophila heat-shock promoter (Fig. 1a). We expected phenotypes similar to those elicited by heatshock induced giant misexpression in Drosophila namely the loss of at least four segments from first instar cuticles [68]. The analysis of heat shocked offspring for cuticle phenotypes did not reveal significant differences to controls that had not been subjected to heat shock (not shown). In conclusion, our attempts to directly transfer Drosophila-based constructs to Tribolium failed.

\section{GAL4 $\triangle$ and GAL4-VP16 activate reporter gene expression via endogenous core promoters}

Core promoters might influence the efficiency of transcription in a species specific way. In order to test if the failure in the above experiments was due to the use of Drosophila core promoters, we made a set of constructs using Tribolium specific core promoters in both driver and responder constructs. We tested two versions of transactivators, GAL4 $\Delta$ and GAL4-VP16 [34,41], both driven by the endogenous heat shock inducible $T c$-hsp 68 promoter element (JBS and GB, unpublished) which con- tains HSF binding sites similar to those found in Drosophila heat inducible promoters (Fig. 1d, e). This promoter leads to strong ubiquitous transcription within $10 \mathrm{~min}$ utes after heat shock in embryos. However, earliest blastoderm stages are refractory to heat shock induced expression while shortly before the morphological differentiation of extraembryonic from embryonic tissue the response is strong (GB, unpublished). The responder, turboGFP (tGFP) was used as reporter, fused to the SV40 early mRNA polyadenylation signal, and driven by UAS sites placed upstream of a $150 \mathrm{bp}$ fragment containing the basal (non-heat-shock-responsive) Tc-hsp68 promoter (Fig. 1k). This core promoter does not drive expression on its own but can do so when combined with enhancer elements (JBS and GB, unpublished). In order to exclude position effects, we analyzed two independent transgenic lines for each construct, i.e. four activator lines were each crossed to two responder lines. Self crossed UAS responders were included as negative controls. A 24 hour egglay was collected and heat shocked (see materials and methods), and then checked for fluorescence every hour. Fastest expression of tGFP in embryos could be observed using the UAS-Tc-bhsp-tGFP\#2 line in combination with both GAL4 $\Delta$ driver lines, while the GAL4-VP16 lines took about one hour longer before tGFP fluorescence could be detected (Table 1). The UAS-Tc-bhsp-tGFP\#7 line tended to be activated later than the \#2 line, indicating some position effect. But also with the \#2 reporter line, GAL4 $\Delta$ tended to perform better than GAL4-VP16. On average, when crossing the two different responder lines to the GAL4 $\Delta$ lines, tGFP fluorescence was visible 3.5 hours after heat shock, whereas in crosses with the GAL4-VP16 lines 4.5 hours were necessary for first detection (Table 1).

\section{GAL4/UAS is widely applicable in different tissues and stages of Tribolium}

In order to test the potential of the GAL4/UAS system for stage or tissue specific expression in Tribolium, we performed heat shocks in larvae, pupae and adult beetles. We observed tGFP fluorescence in animals carrying both the GAL4 $\triangle$ and UAS constructs 24 hours after the heat shock treatment, at all stages tested (Fig. 2d, h), whereas immediately after the heat shock no fluorescence was detected (Fig. 2c, g). As negative controls served pupae and adults carrying both constructs which were not exposed to heat shock (Fig. 2b, f) and heat-shocked adults carrying either the transactivator (not shown) or the responder construct alone (Fig. 2a, e). These controls did not show increased fluorescence compared to wild type (Fig. 2). The same was true for larvae (data not shown). This experiment suggests that the GAL4 $\Delta$ system is active in the beetle at all postembryonic stages. We further analyzed several tissues of adult animals for reporter activity $24 \mathrm{~h}$ after heat shock, and found the system to be 
Table 1: Comparison of GAL4 and GAL4-VP16

\begin{tabular}{lcc}
\hline & \multicolumn{2}{c}{ UAS-Tc-bhsp-tGFP } \\
& \#2 & $\# \mathbf{7}$ \\
\hline Tc-hsp-GAL4 \#1 & $3 \mathrm{~h}$ & $4 \mathrm{~h}$ \\
Tc-hsp-GAL4 \#2 & $3 \mathrm{~h}$ & $4 \mathrm{~h}$ \\
Tc-hsp-GAL4-VP16 \#2 & $4 \mathrm{~h}$ & $4 \mathrm{~h}$ \\
Tc-hsp-GAL4-VP16 \#3 & $4 \mathrm{~h}$ & $6 \mathrm{~h}$
\end{tabular}

Response to GAL4 is slightly faster than to GAL4-VP16. Shown is the time when first fluorescence of tGFP was visible after heat shock. The driver lines Tc-hsp-GAL4 \#1 and \#2 as well as Tc-hsp-GAL4-VP16\#2 and \#3 were crossed against the responder lines UAS-Tc-bhsp-tGFP\#2 and \#7. Combinations containing driver lines based on GAL4 result in earlier responder gene expression ( 3.5 hours on average) than combinations containing GAL4-VP16-based lines ( 4.5 hours on average).

active in all tissues examined including the wings (Fig. 2h), male and female reproductive organs (Fig. $2 \mathrm{~m}$ and $2 q$, respectively), as well as the gut (Fig. $2 u$ ). Because a ubiquitous Gal4 driver is currently not available, we were not able to determine if all cells are responsive to the system. Animals of the same genotype that were not subjected to heat shock did not exhibit fluorescence in any of these tissues (Fig. 2k, o, s).

\section{Endogenous promoters are required for efficient function of transgenes in Tribolium}

These results suggest that the use of endogenous core promoters may be critical for the function of transgenes. To directly compare the relative efficiencies of different core promoters, we crossed the driver line Tc-hspGAL4 $\triangle \# 1$ to different responder lines which were based on non-Tribolium core promoters (Fig. 1f, g, h), induced GAL4 $\Delta$ expression by heat shock and detected the transcript of the reporter gene by whole mount in situ hybridization. To achieve comparable staining levels, all probes were approximately the same size and concentration, and the stainings were developed for the same time. Two independent insertion sites for each responder construct were analyzed to control for integration site effects. As reference we used the above mentioned reporter lines UAS-Tc-bhsp-tGFP\#2 and \#7 were used (Fig. 3a, b).

First, we tested a responder construct containing the Drosophila basal $h s p 70$ promoter (UAS-Dm-hsp-LacZ, Fig. 1f) which has been used successfully in Drosophila to drive $L a c Z$ [23]. We did not detect expression of lacZ mRNA in the offspring (Fig. 3c, d). Next, we tested the SCP1 core promoter which is an artificial core promoter that contains four core promoter motifs - a TATA box from the CytoMegaloVirus (CMV) gene IE1, a initiator (Inr) based on Adenovirus Major Late (AdML) genes and a Drosophila G retrotransposon, a motif ten element (MTE) from Drosophila Tollo and a downstream pro- moter element (DPE) from the Drosophila G core promoter - within a single promoter construct. It directs high levels of transcription by RNA polymerase II in nuclear extracts from Drosophila and HeLa cells, and is more efficient than the CMV or AdML core promoters [69]. This construct (Fig. 1h) contains additional GAGA elements to prevent position effects [70,71]. However, transgenic lines \#F2 and \#M3, carrying the gUAS-SCP1DsRed construct (Fig. 1h), showed no expression of DsRed in embryos when crossed to our driver line and heat shocked (Fig. 3e, f). In order to test whether this failure is Tribolium specific, we generated two independent Drosophila lines using the same gUAS-SCP1-DsRed construct. These lines were crossed to the dpp-GAL4, apGAL4, da-GAL4 and ptc-GAL4 drivers and examined at larval stages. DsRed fluorescence was observed with the dpp-GAL4 driver, roughly consistent with the dpp pattern in discs, but not with the other drivers. Apparently, the vertebrate core promoter gUAS-SCP1 is not working well in insects. Together, these results indicate that neither a core promoter from Drosophila nor an artificial promoter optimized for vertebrate cells is efficient in driving expression in Tribolium.

Assuming that endogenous core promoters are required for efficient transcription, we tested another Tribolium core promoter. The Tc-hairy upstream region has been analyzed previously and from these data the putative Tc-hairy core promoter was deduced [72]. We added this putative basal Tc-hairy promoter $(T c-b h)$ just downstream of the Dm-hsp70TATA in the UAS-Dm-hspLacZ construct and exchanged the reporter $L a c Z$ with egfp. Hence, this construct (UAS-Dm-hsp-Tc-bh-EYFP, Fig. 1i) contains both Drosophila and Tribolium core promoters. When activated by GAL4 $\Delta$, strong expression of eyfp could be detected predominantly within the central nervous system in old embryos (Fig. 3g, h). At earlier stages expression was not efficient (not shown) and the negative control (UAS-Dm-hsp-Tc-bh-EYFP alone) did not exhibit detectable expression of eyfp in the absence of GAL4 transactivator activity (not shown). This surprising expression pattern was identical in two independently generated insertions of the same construct. We confirmed that Tc-hairy is not expressed in the central nervous system in embryos of this developmental stage (not shown). Hence, this unexpected restriction to the nervous system is probably not due to the integration site but either due to a hidden specific activity of the Tc-hairy core promoter, or due to interactions with cryptic binding sites elsewhere in the construct. Thus, although the Tchairy core promoter can confer strong expression in Tribolium, it may not be able to direct expression in all embryonic tissues.

In a similar way we also tested the efficiency of Drosophila promoters in the context of GAL4 drivers, in combination with the responder UAS-Tc-bhsp-tGFP \#2. Dm- 


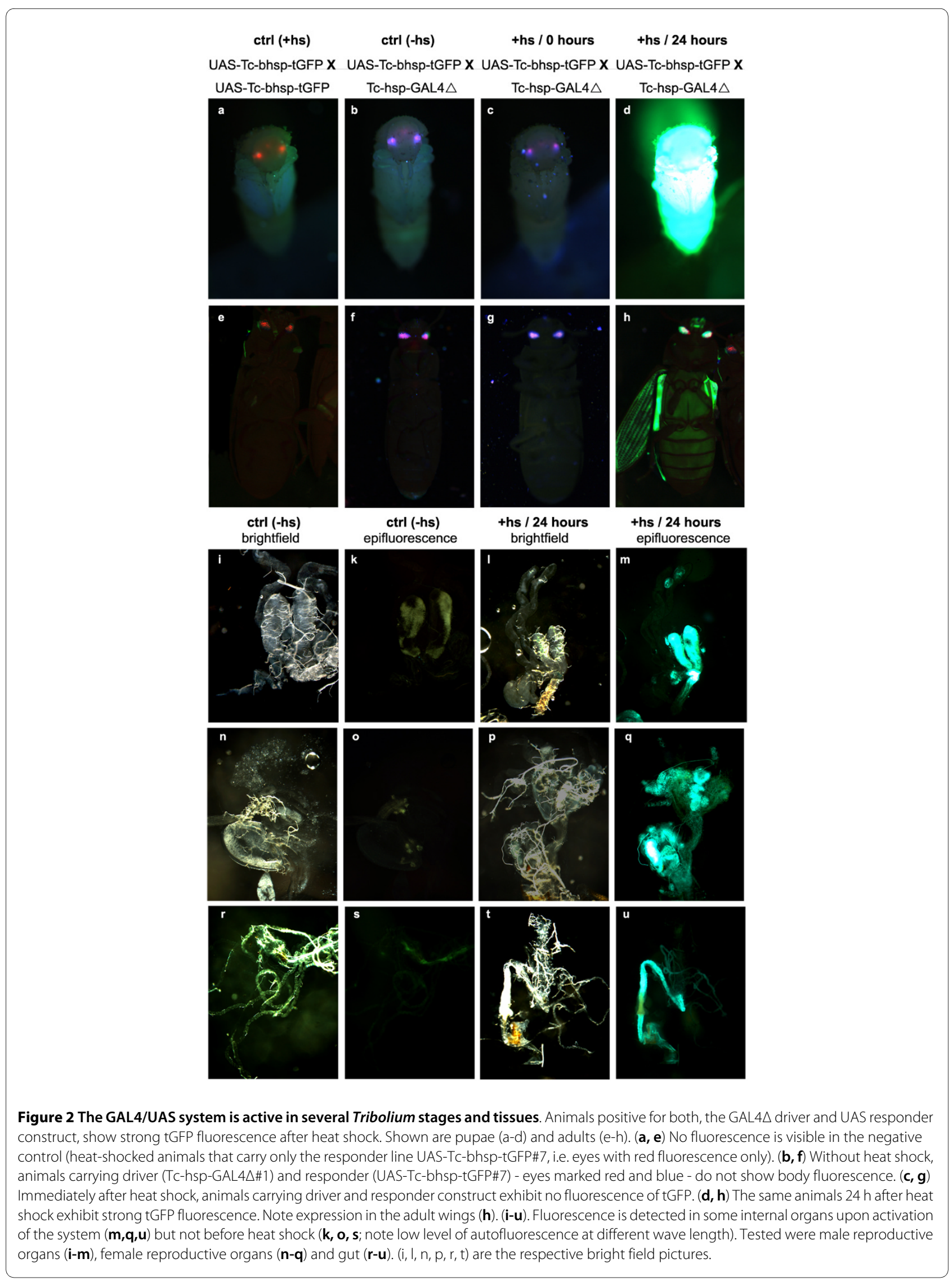




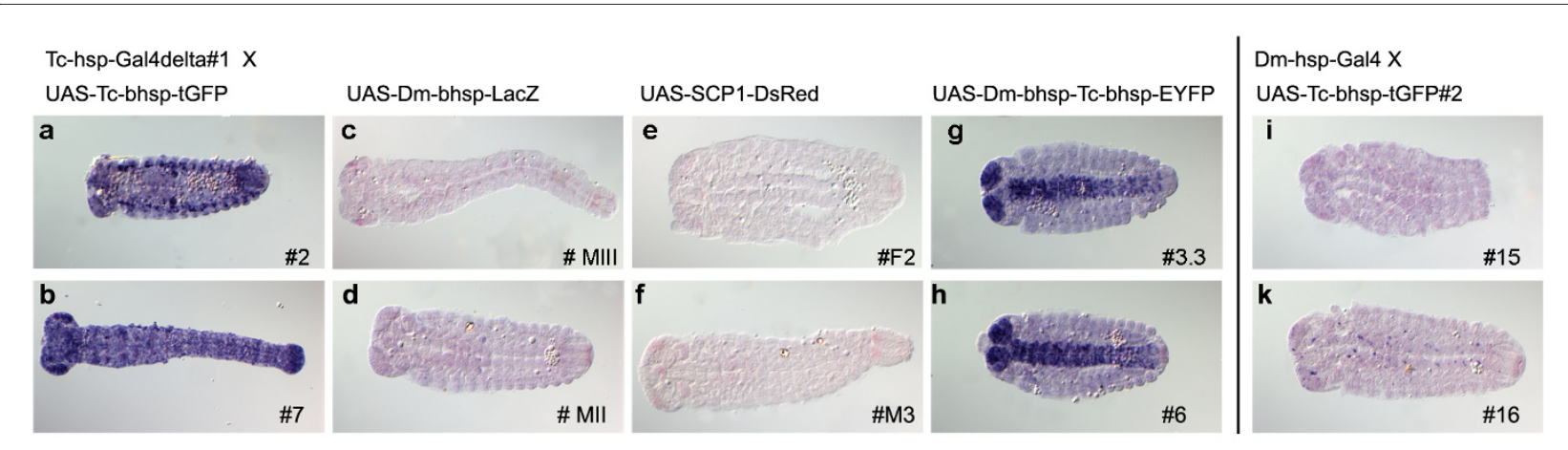

Figure 3 Endogenous versus exogenous promoters in transactivator and responder constructs. Only when Tribolium core promoters are used in the responder and transactivator constructs, activation of the reporter gene is observed via in-situ hybridization. Exogenous promoters are not capable of driving reporter gene expression. (a-h) Transactivator line Tc-hsp-GAL4 $\triangle \# 1$ was crossed to different responder lines and in-situ hybridization was performed with the respective antisense RNA probe. $(\mathbf{a}, \mathbf{b})$ In the positive controls (UAS-Tc-bhsp-tGFP) ubiquitous expression of tGFP is detected. In contrast, no reporter activity is detected in constructs utilizing a Drosophila core promoter (UAS-Dm-hsp-LacZ) (c, d) or an artificial "super core promoter" (UAS-SCP-DsRed) optimized for vertebrate cells $(\mathbf{e}, \mathbf{f})$. $(\mathbf{g}, \mathbf{h})$ A construct containing both the Drosophila basal heat shock promoter and the Tribolium hairy promoter (UAS-Dm-hsp-Tc-bh-EYFP) shows activation predominantly in the nervous system of advanced embryos. (i-k) The Drosophila heat shock promoter driving Gal4 shows no activity in one insertion line (i) while in the other some isolated cells show expression of the reporter (k).

hs-GAL4 (Fig. 1a) utilizes the upstream region of the Drosophila hsp70 gene [73]. These heat shock constructs are frequently used in Drosophila (e.g. [54,74]). The transactivator line Dm-hs-GAL4 \#16 induced weak expression of the reporter gene tGFP in single cells of advanced embryos (Fig. 3k) whereas with the transactivator line Dm-hs-GAL4 \#15 no tGFP expression was detected (Fig. 3i). This indicates that the Drosophila heat shock promoter can elicit only weak Gal4 activity in a subset of Tribolium tissues which in addition may depend on the integration site.

To explore this further, we tested the ability of the Drosophila hsp70 promotor to directly drive EGFP in Tribolium. Studying four independent transgenic lines based on the construct pBac[3XP3-DsRed;Dm-hsp70-EGFP] that showed to be functional in the butterfly Bicyclus anynana [52] we found that the results were highly variable in Tribolium; two lines were capable of activating expression after prolonged heat shock in pupae (1hour at $48^{\circ} \mathrm{C}$ in an incubator; Additional file 1, Fig. S1 B, lines A and E), one was only mildly heat-inducible (Additional file 1, Fig. $\mathrm{S} 1 \mathrm{~B}$, line $\mathrm{B}$ ) and one was active independent of heatshock (Additional file 1, Fig. S1 B; line D). Some of these responses varied across developmental stages (see Additional file 1, Fig. S1 C). Taken together, we find that the Drosphila heat shock promotor does show some activity in Tribolium but its activity is weak and strongly influenced by position effects.

\section{Temperature dependence of the GAL4/UAS system}

In Drosophila, it has been shown that GAL4 activity is enhanced in flies raised at $29^{\circ} \mathrm{C} / 84^{\circ} \mathrm{F}$ compared to lower temperatures [42]. Therefore we wanted to test, whether this temperature dependence exists in Tribolium as well.
0 to 24 hours old Tribolium embryos derived from heterozygous parents carrying the transactivator Tc-hspGAL4 $\triangle \# 1$ and the responder UAS-Tc-bhsp-tGFP\#7 were heat shocked, dechorionated, aligned on a microscope slide and kept at different temperatures. Pictures were taken directly after the heat shock and in one hour intervals to check for the onset of tGFP fluorescence. Indeed we found that higher temperatures lead to an earlier onset of fluorescence ranging from 5 hours $\left(26^{\circ} / 79^{\circ} \mathrm{F}\right)$ to 3-4 hours $\left(32^{\circ} \mathrm{C} / 90^{\circ} \mathrm{F}\right)$ (Fig. 4). However, maximal tGFP activity (24h after heat shock) appears to be similar at both temperatures.

There is little difference in the kinetics of chromophore maturation at $28^{\circ} \mathrm{C}$ and $37^{\circ} \mathrm{C}$ for several GFP variants (this efficiency even decreases for some variants at higher temperatures [75]). Therefore, the earlier onset of tGFP fluorescence at higher temperatures could rather be due to higher GAL4 $\triangle$ activity, due to faster kinetics of the gene regulatory machinery, or both. Tribolium development proceeds twice as fast at $32^{\circ} \mathrm{C}$ versus $26^{\circ} \mathrm{C}$ (total developmental time $6 \mathrm{~d}$ vs. $3 \mathrm{~d}$ ). In these experiments, maximal tGFP levels did not appear grossly different at these temperatures.

\section{The GAL4/UAS system is applicable in Tribolium embryogenesis}

Finally, we analyzed, whether the GAL4/UAS response is sufficiently rapid to analyze gene function during Tribolium embryogenesis. We tested how much time it takes from heat shock to expression of the reporter gene using the best combination of driver and responder lines (Tchsp-GAL4 $\triangle \# 1$ and UAS-Tc-bhsp-tGFP \#7). A one hour egglay was collected and embryos were allowed to develop further until the end of germ band elongation. 


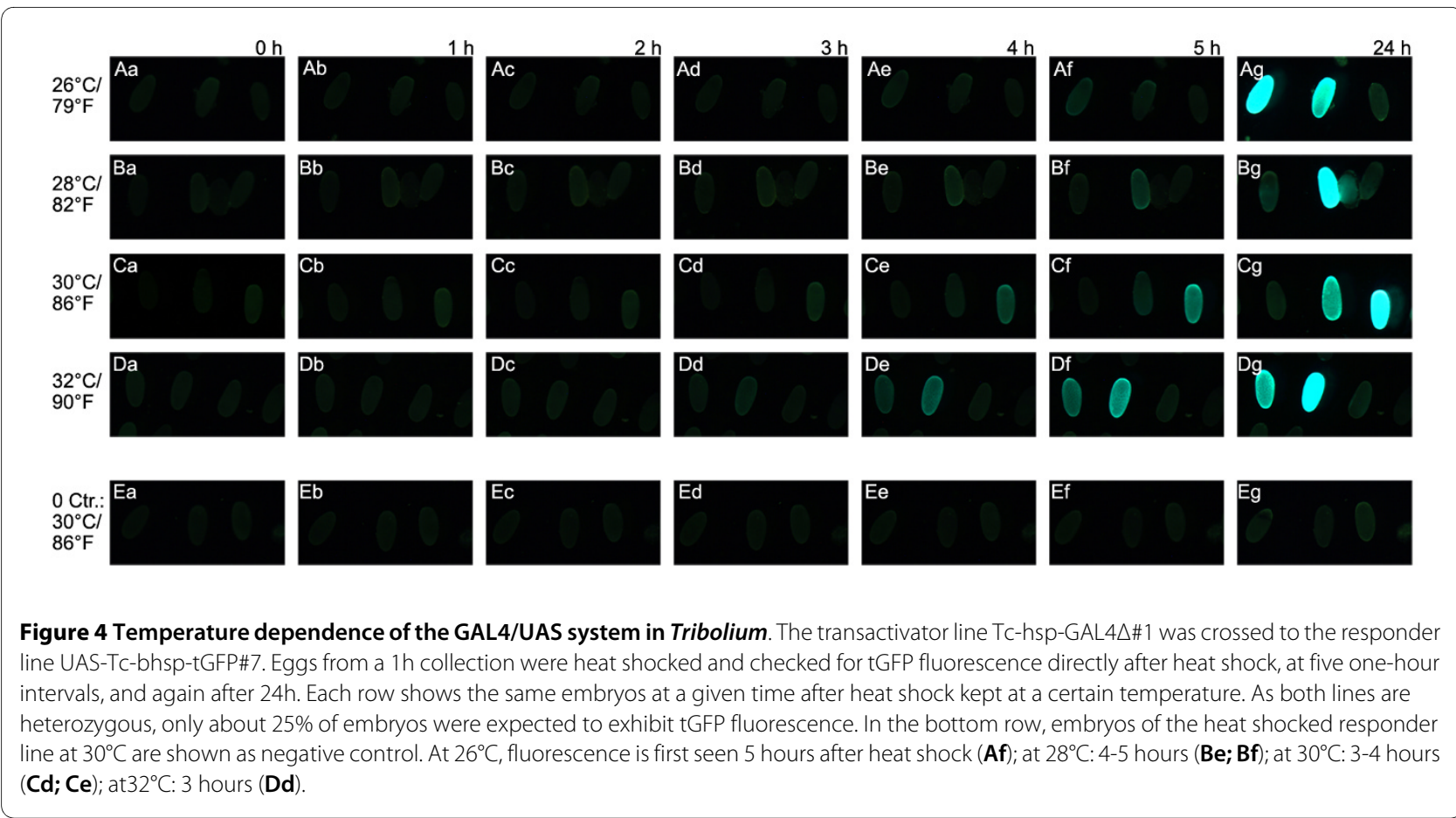

The embryos were then heat shocked and fixed for in-situ hybridization at different time points after the heat shock. It was unclear how temperature would affect the system - on the one hand embryonic development is about twice as fast at $32^{\circ} \mathrm{C}$ compared to $25^{\circ} \mathrm{C}$, on the other hand the heat shock may slow down development and recovery might be aided by lower temperature. Hence, treated embryos were kept at $32^{\circ} \mathrm{C}$ or $25^{\circ} \mathrm{C}$ after heat shock. Embryos kept at $25^{\circ} \mathrm{C}$ were fixed immediately, or two, four and six hours after heat shock treatment (Fig. 5a-d). Embryos kept at $32^{\circ} \mathrm{C}$ were fixed directly after heat shock and after two, three and four hours, because faster onset was expected at higher temperatures (see above) (Fig. 5n-q). tGFP expression was detected via in-situ hybridization as an immediate readout of gene expression.

Earliest tGFP expression could be observed four hours after heat shock $\left(10 \mathrm{~min}\right.$ at $\left.46^{\circ} \mathrm{C}\right)$ when incubated at $25^{\circ} \mathrm{C}$, whereas embryos kept at $32^{\circ} \mathrm{C}$ after heat shock showed expression already after two hours (compare Fig. 5c to 5o). Strong expression was detectable already after three hours at $32^{\circ} \mathrm{C}$ while six hours were required for a similar expression level at $25^{\circ} \mathrm{C}$ (compare Fig. $5 \mathrm{~d}$ to $5 \mathrm{p}$ ). At the stages tested, the morphology of the head and the elongation of appendages provide a good measure for the age of the embryo. We compared overall morphology, head morphology and the length of the trunk appendages at the time of heat shock (Fig. 5e, i and 5r, v) and when the reporter was fully expressed (Fig. $5 \mathrm{~h}, \mathrm{~m}$ and $5 \mathrm{t}$, $\mathrm{x}$, respectively) in at least 5 embryos per time point. We do not find a major difference indicating that the target gene response is fast relative to developmental time.

\section{Discussion}

We have adopted the GAL4/UAS system to Tribolium and find that GAL4 $\Delta[33,34]$ is slightly superior to GAL4VP16 [41]. This came not unexpected as GAL4 $\Delta$ is a much smaller protein that consists only of the DNA binding and activation domains of GAL4. As potential toxic effects of GAL4-VP16 have been observed for Drosophila $[23,76]$ and in other organisms $[41,77]$ we suggest the use of GAL $4 \Delta$ in Tribolium in the future.

The GAL4 $\Delta$ /UAS system is active at all stages of Tribolium development and activates reporter gene expression in a variety of different tissues indicating broad applicability. Interestingly, the activation of a UAS target is relatively fast compared to embryonic development which will allow the use of the system to investigate embryogenesis in addition to postembryonic stages. In fact, development does not seem to proceed much after heat shock (compare embryos $\mathrm{d}$ to a and $\mathrm{q}$ to $\mathrm{n}$ in Fig. 5). This is in line with findings in Drosophila where a $15^{\prime}$ arrest of development has been observed upon heat shock [78]. Like in Drosophila, Tribolium embryos resume development after some time and do not show elevated lethality or cuticle phenotypes due to the heat shock conditions used (not shown). In contrast, the extremely fast mode of Drosophila early development has in many cases hampered the use of the Gal4/UAS system in the study of some processes. Furthermore, Gal4 is likely to be fully active at the temperatures commonly used to raise Tribo- 


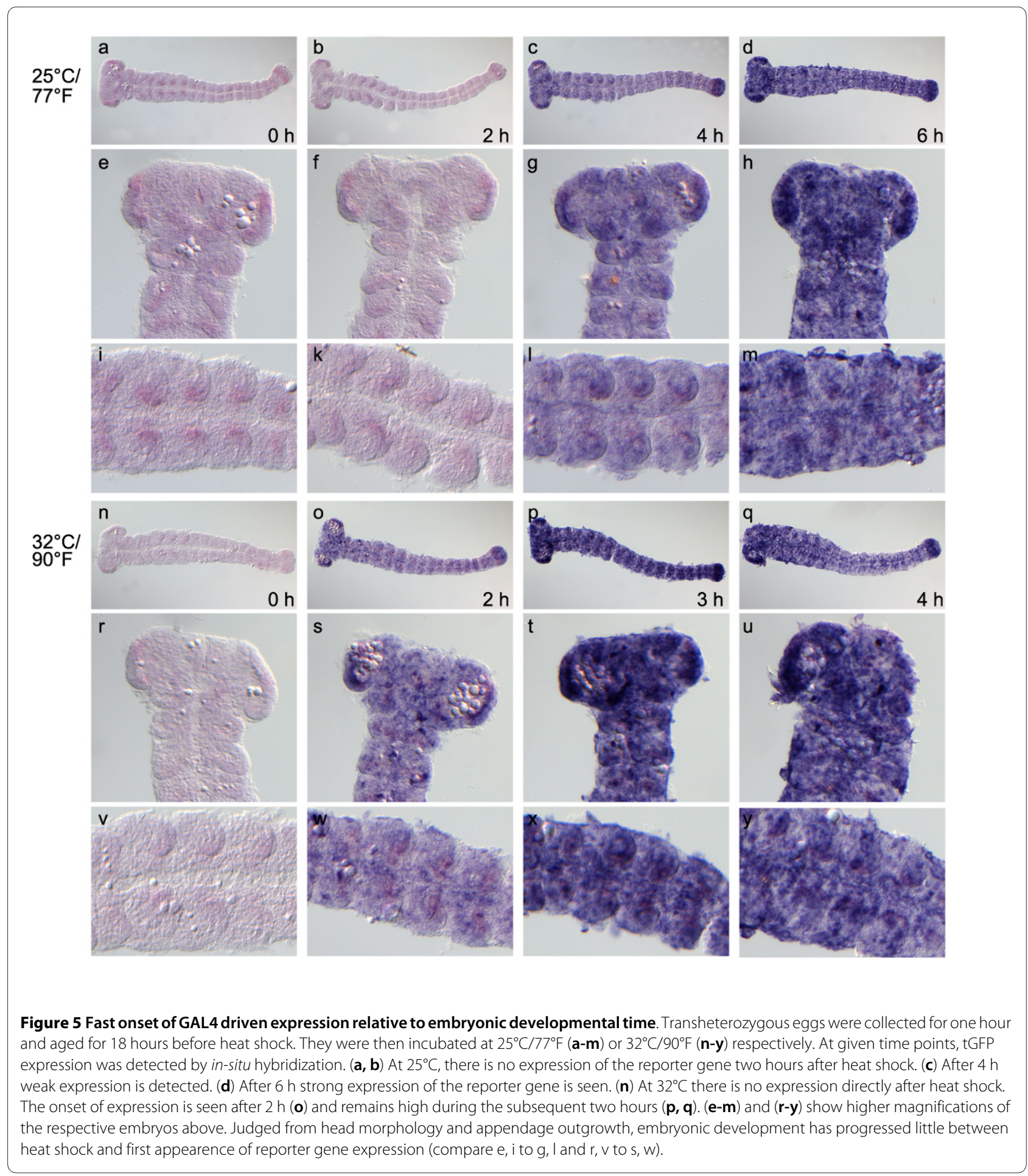

lium $\left(25-32^{\circ} \mathrm{C}\right)$ because they are similar to the temperature optimum for yeast $\left(25-30^{\circ} \mathrm{C}\right)$ [79].

In contrast to endogenous core promoters, neither the Drosophila heat shock core promoter nor an artificial "super core promoter" (SCP1) consisting of optimized vertebrate core promoter motifs were effective in driving expression in Tribolium. Similar experience has recently been described for the tephritid fruit fly Ceratitis capitata $[80,81]$. On the other hand, Drosophila heat shock constructs have been shown to work in the silkworm Bombyx mori [46] and the butterfly Bicyclus anynana [52]. Moreover, the artificial 3xP3 enhancer is driving flu- 
orescent reporters in the Tribolium eyes and brain using a Drosophila core promoter [5], and the same is true in a wide variety of other organisms [50,61-66]. In the light of our results it appears that the 3XP3 element is extremely efficient in driving expression and hence is able to override the poor function of the exogenous core promoter.

\section{Conclusions}

The establishment of the Gal4/UAS system in Tribolium allows more profound functional gene analysis by directed expression in this species in the future. This will further promote Tribolium as a model organism where general biological questions can be studied. To take full benefit of the system, it is essential to generate a collection of driver lines that allow misexpression in different tissues. To this end it will be expedient to perform random insertion screens with GAL4 $\Delta$-containing mutator constructs following a procedure similar to the one recently used in the large scale GEKU insertional mutagenesis screen [10].

One important lesson for future transgenic tools in Tribolium and probably also other species is that the use of endogenous promoters is necessary for efficient expression. Even if constructs based on exogenous core promoters show some activity under certain circumstances or in some species, for full functionality, the use of speciesspecific promoters is essential.

\section{Additional material}

Additional file 1 Drosophila promoter does not work reliably in Tribo-
lium. A) Variants of Drosophila-based Gal4/UAS systems do not show
any activity in Tribolium castaneum. UAS-dependent LacZ driven by a
Drosophila core promoter (UAST) does not lead to detectable protein
expression in adult heads (lanes $1 \mathrm{~h} / 2 \mathrm{~h}$, ten heads, respectively) or abdo-
men ( $1 \mathrm{a} / 2 \mathrm{a}$, one abdomen, respectively), when activated by 3xP3-driven
GAL4 $\triangle$ (lanes $1 \mathrm{~h} / 1 \mathrm{a}$ ) or Gal4-VP16 (lanes $2 \mathrm{~h} / 2 \mathrm{a}$ ). Negative controls: UAST
responder alone (lane 3 ) and vermilionwhite strain without transgenes (lane
4). Functionality of the anti-ß-galactosidase antibody was confirmed by an
extract of Drosophila heads expressing lacZ by a functional LexAGAD/(LL)4
system ([23]; 3 heads used, lane 5 ). Additionally, reprobing of the blot with
an anti-alpha-tubulin antibody was performed as a loading control. B) The
inducibility of the Drosophila heat shock promoter is low in Tribolium
and its activity subject to position effect. Four independent insertions of
a construct with the Drosophila heat shock 70 promoter driving EGFP [52]
were tested at the pupal stage (lines A, B, D and E). "+hs" indicates heat
shocked animals. As controls, transheterozygotes without heatshock (-hs)
and a heatshocked wt control (wt +hs) were included. Line A and E showed
some activation at the pupal stage while little activation was observed in
lines B and D. Moreover, line D showed some constitutive activity. C) The
inducibility of the Drosophila heat shock promoter is unreliable and
partially stage-dependent in Tribolium. Heat shock activation of Droso-
phila heat shock 70 promoter driving EGFP [52] line A is strong only at the
pupal stage. "+hs" indicates heat shocked animals. As controls, transhet-
erozygotes without heatshock (-hs) and a heatshocked wt control (wt +hs)
were included.

\section{Authors' contributions}

JBS made the constructs and the experiments using the endogenous Tribolium promoters and wrote the paper. GB designed this part of the study and wrote the paper. Constructs based on Drosophila core promoters and their tests in Tribolium were done by IV, MW, and GB under the guidance of EAW and MK. The
gUAS-SCP1 construct and transgenic lines were contributed by AK and MA. All authors read and approved of the final version of the paper.

\section{Acknowledgements}

We thank Xiaohui Zeng for help with one construct. This work was supported by the DFG (BU1443/3-1 to G.B. and DFG-Graduiertenkolleg 678 Ph.D. stipend to I.V.), by the HFSPO (RG 303 to M.K.), the Robert Bosch Foundation (E.A.W.) and the Marie Curie RTN programme 'ZOONET' (to M.A. and E.A.W.).

\section{Author Details}

1 Ernst Caspari Haus, Georg-August-University Göttingen, Justus-von-LiebigWeg11, 37077 Göttingen, Germany, 2Department of Biology, FriedrichAlexander-University Erlangen, Staudtstr. 5, 91058 Erlangen, Germany, 3Institute of Molecular Biology and Biotechnology (IMBB), Foundation for Research and Technology Hellas (FoRTH), GR-70013 Iraklio Crete, Greece, 4 Max Planck Institute of Molecular Cell Biology and Genetics, Pfotenhauerstr. 108, 01307 Dresden, Germany and ${ }^{5}$ Centre for Organismal Systems Biology, Faculty of Life Sciences, University of Vienna, Althanstrasse 14, 1090 Wien, Austria

Received: 25 January 2010 Accepted: 19 May 2010

Published: 19 May 2010

\section{References}

1. Hunt T, Bergsten J, Levkanicova Z, Papadopoulou A, John OS, Wild R, Hammond PM, Ahrens D, Balke M, Caterino MS, et al: A comprehensive phylogeny of beetles reveals the evolutionary origins of a superradiation. Science 2007, 318:1913-6.

2. Klingler M: Tribolium. Curr Biol 2004, 14:R639-40.

3. Schroder R, Beermann A, Wittkopp N, Lutz R: From development to biodiversity--Tribolium castaneum, an insect model organism for short germband development. Dev Genes Evol 2008, 218:119-26.

4. Richards S, Gibbs RA, Weinstock GM, Brown SJ, Denell R, Beeman RW, Gibbs R, Beeman RW, Brown SJ, Bucher G, et al:: The genome of the model beetle and pest Tribolium castaneum. Nature 2008, 452:949-55.

5. Berghammer AJ, Klingler M, Wimmer EA: A universal marker for transgenic insects. Nature 1999, 402:370-1.

6. Horn C, Schmid BG, Pogoda FS, Wimmer EA: Fluorescent transformation markers for insect transgenesis. Insect Biochem Mol Biol 2002, 32:1221-35.

7. Lorenzen MD, Berghammer AJ, Brown SJ, Denell RE, Klingler M, Beeman RW: piggyBac-mediated germline transformation in the beetle Tribolium castaneum. Insect Mol Biol 2003, 12:433-40.

8. Pavlopoulos A, Berghammer AJ, Averof M, Klingler M: Efficient transformation of the beetle Tribolium castaneum using the Minos transposable element: quantitative and qualitative analysis of genomic integration events. Genetics 2004, 167:737-46.

9. Lorenzen MD, Kimzey T, Shippy TD, Brown SJ, Denell RE, Beeman RW: piggyBac-based insertional mutagenesis in Tribolium castaneum using donor/helper hybrids. Insect Mol Biol 2007, 16:265-75.

10. Trauner J, Schinko J, Lorenzen MD, Shippy TD, Wimmer EA, Beeman RW, Klingler M, Bucher G, Brown SJ: Large-scale insertional mutagenesis of a coleopteran stored grain pest, the red flour beetle Tribolium castaneum, identifies embryonic lethal mutations and enhancer traps. BMC Biol 2009, 7:73.

11. Brown SJ, Mahaffey JP, Lorenzen MD, Denell RE, Mahaffey JW: Using RNAi to investigate orthologous homeotic gene function during development of distantly related insects. Evol Dev 1999, 1:11-5.

12. Bucher G, Scholten J, Klingler M: Parental RNAi in Tribolium (Coleoptera). Curr Biol 2002, 12:R85-6.

13. Tomoyasu Y, Denell RE: Larval RNAi in Tribolium (Coleoptera) for analyzing adult development. Dev Genes Evol 2004, 214:575-8.

14. Miller SC, Brown SJ, Tomoyasu Y: Larval RNAi in Drosophila? Dev Genes Evol 2008, 218:505-10.

15. Konopova B, Jindra M: Juvenile hormone resistance gene Methoprenetolerant controls entry into metamorphosis in the beetle Tribolium castaneum. Proc Natl Acad Sci USA 2007, 104:10488-93.

16. Konopova B, Jindra M: Broad-Complex acts downstream of Met in juvenile hormone signaling to coordinate primitive holometabolan metamorphosis. Development 2008, 135:559-68.

17. Suzuki Y, Squires DC, Riddiford LM: Larval leg integrity is maintained by Distal-less and is required for proper timing of metamorphosis in the flour beetle, Tribolium castaneum. Dev Biol 2009, 326:60-7. 
18. Tomoyasu Y, Miller SC, Tomita S, Schoppmeier M, Grossmann D, Bucher G: Exploring systemic RNA interference in insects: a genome-wide survey for RNAi genes in Tribolium. Genome Biol 2008, 9:R10.

19. Cerny AC, Grossmann D, Bucher G, Klingler M: The Tribolium ortholog of knirps and knirps-related is crucial for head segmentation but plays a minor role during abdominal patterning. Dev Biol 2008, 321:284-94

20. Brand $\mathrm{AH}$, Perrimon $\mathrm{N}$ : Targeted gene expression as a means of altering cell fates and generating dominant phenotypes. Development 1993, 118:401-15

21. Bello B, Resendez-Perez D, Gehring WJ: Spatial and temporal targeting of gene expression in Drosophila by means of a tetracycline-dependent transactivator system. Development 1998, 125:2193-202.

22. Stebbins MJ, Urlinger S, Byrne G, Bello B, Hillen W, JC Yin: Tetracyclineinducible systems for Drosophila. Proc Natl Acad Sci USA 2001, 98:10775-80.

23. Viktorinova I, Wimmer EA: Comparative analysis of binary expression systems for directed gene expression in transgenic insects. Insect Biochem Mol Biol 2007, 37:246-54.

24. McGuire SE, Roman G, Davis RL: Gene expression systems in Drosophila: a synthesis of time and space. Trends Genet 2004, 20:384-91.

25. Szuts D, Bienz M: LexA chimeras reveal the function of Drosophila Fos as a context-dependent transcriptional activator. Proc Natl Acad Sci USA 2000, 97:5351-6.

26. Zhong J, Yedvobnick B: Targeted gain-of-function screening in Drosophila using GAL4-UAS and random transposon insertions. Genet Res 2009, 91:243-58

27. Fischer JA, Giniger E, Maniatis T, Ptashne M: GAL4 activates transcription in Drosophila. Nature 1988, 332:853-6.

28. Rorth P: Gal4 in the Drosophila female germline. Mech Dev 1998, 78:113-8

29. Ornitz DM, Moreadith RW, Leder P: Binary system for regulating transgene expression in mice: targeting int-2 gene expression with yeast GAL4/UAS control elements. Proc Natl Acad Sci USA 1991, 88:698-702.

30. Laughon A, Driscoll R, Wills N, Gesteland RF: Identification of two proteins encoded by the Saccharomyces cerevisiae GAL4 gene. Mol Cell Biol 1984, 4:268-75.

31. Laughon A, Gesteland RF: Primary structure of the Saccharomyces cerevisiae GAL4 gene. Mol Cell Biol 1984, 4:260-7.

32. Giniger E, Varnum SM, Ptashne M: Specific DNA binding of GAL4, a positive regulatory protein of yeast. Cell 1985, 40:767-74.

33. Horn C, Offen N, Nystedt S, Hacker U, Wimmer EA: piggyBac-based insertional mutagenesis and enhancer detection as a tool for functional insect genomics. Genetics 2003, 163:647-61.

34. Ma J, Ptashne M: Deletion analysis of GAL4 defines two transcriptional activating segments. Cell 1987, 48:847-53.

35. Triezenberg SJ, Kingsbury RC, McKnight SL: Functional dissection of VP16, the trans-activator of herpes simplex virus immediate early gene expression. Genes Dev 1988, 2:718-29.

36. Triezenberg SJ, LaMarco KL, MCKnight SL: Evidence of DNA: protein interactions that mediate HSV-1 immediate early gene activation by VP16. Genes Dev 1988, 2:730-42.

37. O'Hare P, Goding CR: Herpes simplex virus regulatory elements and the immunoglobulin octamer domain bind a common factor and are both targets for virion transactivation. Cell 1988, 52:435-45.

38. O'Hare P, Goding CR, Haigh A: Direct combinatorial interaction between a herpes simplex virus regulatory protein and a cellular octamerbinding factor mediates specific induction of virus immediate-early gene expression. Embo J 1988, 7:4231-8.

39. Preston CM, Frame MC, Campbell ME: A complex formed between cell components and an HSV structural polypeptide binds to a viral immediate early gene regulatory DNA sequence. Cell 1988, 52:425-34.

40. McKnight JL, Kristie TM, Roizman B: Binding of the virion protein mediating alpha gene induction in herpes simplex virus 1-infected cells to its cis site requires cellular proteins. Proc Natl Acad Sci USA 1987, 84:7061-5.

41. Sadowski I, Ma J, Triezenberg S, Ptashne M: GAL4-VP16 is an unusually potent transcriptional activator. Nature 1988, 335:563-4

42. Brand AH, Manoukian AS, Perrimon N: Ectopic expression in Drosophila. Methods Cell Biol 1994, 44:635-54.

43. Duffy JB: GAL4 system in Drosophila: a fly geneticist's Swiss army knife. Genesis 2002, 34:1-15.
44. McGuire SE, Mao Z, Davis RL: Spatiotemporal gene expression targeting with the TARGET and gene-switch systems in Drosophila. SciSTKE 2004, 2004:pl6.

45. Ma J, Ptashne M: The carboxy-terminal 30 amino acids of GAL4 are recognized by GAL80. Cell 1987, 50:137-42.

46. Imamura M, Nakai J, Inoue S, Quan GX, Kanda T, Tamura T: Targeted gene expression using the GAL4/UAS system in the silkworm Bombyx mori. Genetics 2003, 165:1329-40.

47. Scheer N, Campos-Ortega JA: Use of the Gal4-UAS technique for targeted gene expression in the zebrafish. Mech Dev 1999, 80:153-8.

48. Hartley KO, Nutt SL, Amaya E: Targeted gene expression in transgenic Xenopus using the binary Gal4-UAS system. Proc Natl Acad Sci USA 2002, 99:1377-82

49. Guyer D, Tuttle A, Rouse S, Volrath S, Johnson M, Potter S, Gorlach J, Goff S, Crossland L, Ward E: Activation of latent transgenes in Arabidopsis using a hybrid transcription factor. Genetics 1998, 149:633-9.

50. Horn C, Wimmer EA: A versatile vector set for animal transgenesis. Dev Genes Evol 2000, 210:630-7.

51. Li X, Harrell RA, Handler AM, Beam T, Hennessy K, Fraser MJ Jr: piggyBac internal sequences are necessary for efficient transformation of target genomes. Insect Mol Biol 2005, 14:17-30.

52. Ramos DM, Kamal F, Wimmer EA, Cartwright AN, Monteiro A: Temporal and spatial control of transgene expression using laser induction of the hsp70 promoter. BMC Dev Biol 2006, 6:55.

53. Berghammer AJ, Weber M, Trauner J, Klingler M: Red Flour Beetle (Tribolium) Germline Transformation and Insertional Mutagenesis. Cold Spring Harbor Protocols 2009.

54. Handler AM, Harrell RA: Germline transformation of Drosophila melanogaster with the piggyBac transposon vector. Insect Mol Biol 1999, 8:449-57.

55. Cormack BP, Valdivia RH, Falkow S: FACS-optimized mutants of the green fluorescent protein (GFP). Gene 1996, 173:33-8.

56. Yang TT, Cheng L, Kain SR: Optimized codon usage and chromophore mutations provide enhanced sensitivity with the green fluorescent protein. Nucleic Acids Res 1996, 24:4592-3.

57. Cubitt $A B$, Woollenweber $L A$, Heim R: Understanding structure-function relationships in the Aequorea victoria green fluorescent protein. Methods Cell Biol 1999, 58:19-30.

58. Patterson G, Day RN, Piston D: Fluorescent protein spectra. J Cell Sci 2001, 114:837-8

59. Schinko J, Posnien N, Kittelmann S, Koniszewski N, Bucher G: Single and Double Whole-Mount In Situ Hybridization in Red Flour Beetle (Tribolium) Embryos. Cold Spring Harbor Protocols 2009. pdb.prot5258

60. Horn C, Jaunich B, Wimmer EA: Highly sensitive, fluorescent transformation marker for Drosophila transgenesis. Dev Genes Evol 2000, 210:623-9.

61. Kokoza V, Ahmed A, Wimmer EA, Raikhel AS: Efficient transformation of the yellow fever mosquito Aedes aegypti using the piggyBac transposable element vector pBac[3xP3-EGFP afm]. Insect Biochem Mol Biol 2001, 31:1137-43.

62. Scolari F, Schetelig MF, Bertin S, Malacrida AR, Gasperi G, Wimmer EA: Fluorescent sperm marking to improve the fight against the pest insect Ceratitis capitata (Wiedemann; Diptera: Tephritidae). N Biotechnol 2008, 25:76-84

63. Pavlopoulos A, Averof M: Establishing genetic transformation for comparative developmental studies in the crustacean Parhyale hawaiensis. Proc Natl Acad Sci USA 2005, 102:7888-93.

64. Marcus JM, Ramos DM, Monteiro A: Germline transformation of the butterfly Bicyclus anynana. Proc Biol Sci 2004, 271(Suppl 5):S263-5.

65. Thomas JL, Da Rocha M, Besse A, Mauchamp B, Chavancy G: 3xP3-EGFP marker facilitates screening for transgenic silkworm Bombyx mori L. from the embryonic stage onwards. Insect Biochem Mol Biol 2002, 32:247-53.

66. Kuwayama H, Yaginuma T, Yamashita O, Niimi T: Germ-line transformation and RNAi of the ladybird beetle, Harmonia axyridis. Insect Mol Biol 2006, 15:507-12.

67. Bucher G, Klingler M: Divergent segmentation mechanism in the short germ insect Tribolium revealed by giant expression and function. Development 2004, 131:1729-40.

68. Kraut R, Levine M: Mutually repressive interactions between the gap genes giant and Kruppel define middle body regions of the Drosophila embryo. Development 1991, 111:611-21. 
69. Juven-Gershon T, Cheng S, Kadonaga JT: Rational design of a super core promoter that enhances gene expression. Nat Methods 2006, 3:917-22.

70. Tsukiyama T, Becker PB, Wu C: ATP-dependent nucleosome disruption at a heat-shock promoter mediated by binding of GAGA transcription factor. Nature 1994, 367:525-32.

71. O'Donnell KH, Chen CT, Wensink PC: Insulating DNA directs ubiquitous transcription of the Drosophila melanogaster alpha 1-tubulin gene. Mol Cell Biol 1994, 14:6398-408.

72. Eckert C, Aranda M, Wolff C, Tautz D: Separable stripe enhancer elements for the pair-rule gene hairy in the beetle Tribolium. EMBO Rep 2004, 5:638-42.

73. Lis JT, Simon JA, Sutton CA: New heat shock puffs and betagalactosidase activity resulting from transformation of Drosophila with an hsp70-lacZ hybrid gene. Cell 1983, 35:403-10.

74. Certel SJ, Johnson WA: Disruption of mesectodermal lineages by temporal misexpression of the Drosophila POU-domain transcription factor, drifter. Dev Genet 1996, 18:279-88.

75. Patterson GH, Knobel SM, Sharif WD, Kain SR, Piston DW: Use of the green fluorescent protein and its mutants in quantitative fluorescence microscopy. Biophys J 1997, 73:2782-90.

76. Driever W, Ma J, Nusslein-Volhard C, Ptashne M: Rescue of bicoid mutant Drosophila embryos by bicoid fusion proteins containing heterologous activating sequences. Nature 1989, 342:149-54.

77. Gill G, Ptashne M: Negative effect of the transcriptional activator GAL4. Nature 1988, 334:721-4.

78. Ish-Horowicz D, Pinchin SM: Pattern abnormalities induced by ectopic expression of the Drosophila gene hairy are associated with repression of ftz transcription. Cell 1987, 51:405-15.

79. Barnett JA, Payne RW, Yarrow D: Yeasts: Characteristics and Identification. 1st edition. Cambridge: Cambridge University Press; 1983.

80. Kalosaka K, Chrysanthis G, Rojas-Gill AP, Theodoraki M, Gourzi P, Kyriakopoulos A, Tatari M, Zacharopoulou A, Mintzas AC: Evaluation of the activities of the medfly and Drosophila hsp70 promoters in vivo in germ-line transformed medflies. Insect Mol Biol 2006, 15:373-82.

81. Schetelig MF, Caceres C, Zacharopoulou A, Franz G, Wimmer EA

Conditional embryonic lethality to improve the sterile insect technique in Ceratitis capitata (Diptera: Tephritidae). BMC Bio/ 2009, 7:4

doi: $10.1186 / 1471-213 X-10-53$

Cite this article as: Schinko et al., Functionality of the GAL4/UAS system in Tribolium requires the use of endogenous core promoters BMC Developmental Biology 2010, 10:53

Submit your next manuscript to BioMed Centra and take full advantage of:

- Convenient online submission

- Thorough peer review

- No space constraints or color figure charges

- Immediate publication on acceptance

- Inclusion in PubMed, CAS, Scopus and Google Scholar

- Research which is freely available for redistribution

Submit your manuscript at www.biomedcentral.com/submit
C Biomed Central 